\title{
Sunscreens and Photoaging: A Review of Current Literature
}

\author{
Linna L. Guan ${ }^{1} \cdot$ Henry W. Lim ${ }^{1} \cdot$ Tasneem F. Mohammad $^{1}$ \\ Accepted: 29 July 2021 / Published online: 13 August 2021 \\ (c) The Author(s), under exclusive licence to Springer Nature Switzerland AG 2021
}

\begin{abstract}
Sunscreens have been on the market for many decades as a means of protection against ultraviolet-induced erythema. Over the years, evidence has also shown their efficacy in the prevention of photoaging, dyspigmentation, DNA damage, and photocarcinogenesis. In the USA, most broad-spectrum sunscreens provide protection against ultraviolet B (UVB) radiation and short-wavelength ultraviolet A (UVA) radiation. Evidence suggests that visible light and infrared light may play a role in photoaging and should be considered when choosing a sunscreen. Currently, there is a paucity of US FDA-approved filters that provide protection against long UVA $(>370 \mathrm{~nm})$ and none against visible light. Additionally, various sunscreen additives such as antioxidants and photolyases have also been reported to protect against and possibly reverse signs of photoaging. This literature review evaluates the utility of sunscreen in protecting against photoaging and further explores the requirements for an ideal sunscreen.
\end{abstract}

\section{Key Points}

The perception of sunscreen use has shifted from purely protecting against ultraviolet (UV)-induced erythema to broad-spectrum protection against not only erythema but also photoaging, dyspigmentation, DNA damage, and photocarcinogenesis.

Evidence suggests that visible light and infrared light may play a role in photoaging and should be considered when choosing a sunscreen. A broad-spectrum tinted sunscreen with sun protection factor (SPF) $\geq 30$ used daily will offer protection against UV radiation and visible light to reduce their effects on photoaging.

Sunscreen additives such as antioxidants, photolyases, and more have not only opened the door to improved photoprotection against skin aging but also the exploration of newer theories in the reversal of skin aging, but larger-scale and replicable studies are needed before clinical guidelines can be issued.

Tasneem F. Mohammad

tmohamm2@hfhs.org

1 Department of Dermatology, Henry Ford Health Systems, Henry Ford Medical Center-New Center One, 3031 W. Grand Boulevard, Suite 800, Detroit, MI 48202, USA

\section{Introduction}

Chronic sun exposure has long been known to cause photoaging, a process where the skin undergoes changes in epidermal thickness, increases in pigment heterogeneity and dermal elastosis, degradation of collagen in the dermis, development of ectatic vessels, and increases in mutagenesis of keratinocytes and melanocytes in the skin [1]. Clinically, this is characterized by an increase in rhytides, telangiectasias, dyspigmentation including lentigines and ephelides, volume loss, and cutaneous malignancies [1]. A recent observational study further characterized skin aging as hypertrophic and atrophic variants, with atrophic photoaging presenting with erythema and increased risk of skin cancers and hypertrophic photoaging with increased skin thickness and sallowness [2].

In today's society, the value placed on a youthful appearance is reflected in the multibillion-dollar industry centered around anti-aging products [3, 4]. It has been reported that approximately $80 \%$ of skin aging on the face can be attributed to ultraviolet (UV) exposure [5]. Therefore, despite the emphasis of the market on the reversal of skin aging, the best defense against cutaneous age-related changes is through prevention with rigorous photoprotection [4]. It should be noted that proper photoprotection consists of seeking shade when outdoors; wearing a widebrimmed hat, photoprotective clothing, and sunglasses; and applying sun protection factor (SPF) $\geq 30$ broadspectrum tinted sunscreen on exposed sites. 
In the USA, most broad-spectrum sunscreens provide protection against UVB radiation and short wavelength UVA radiation. However, there is a paucity of US FDAapproved filters that provide protection against long UVA $(>370 \mathrm{~nm})$ and none against visible light (VL), making the ideal sunscreen a product that requires further innovation and research. Notable exceptions are pigmentary grade zinc oxide and titanium dioxide, which reflect VL; however, the whitish discoloration they leave on the surface of the skin makes them cosmetically unappealing to consumers. This review evaluates the utility of sunscreen in protecting against photoaging and further explores the requirements of an ideal sunscreen.

\section{Electromagnetic Radiation and Photoaging}

Solar UV radiation (UVR) consists of UVA (320-400 nm), UVB (280-320 nm), and UVC (100-280 nm). UVA is further categorized as UVA1 $(340-400 \mathrm{~nm})$ and UVA2 $(320-340 \mathrm{~nm})$. UVC is the shortest wavelength and considered the most damaging type of UVR. However, it is completely absorbed by the ozone and does not reach the earth's surface [6].

UVB is the major portion of UVR that induces sunburns or UV-induced erythema. It is known to be significantly more erythemogenic than UVA [6]. For example, for skin phototype I, the minimal erythema dose for UVB is $20-40$ $\mathrm{mJ} / \mathrm{cm}^{2}$, whereas that for UVA is $20-40 \mathrm{~J} / \mathrm{cm}^{2}$. Although UVB accounts for approximately $6 \%$ of all UVR that reaches the earth's surface, it is more cytotoxic than UVA, causing direct DNA damage through photon absorption in the form of cyclobutane pyrimidine dimers (CPDs) or 6,4-photoproducts that eventually induce mutagenesis and skin cancers $[7$, 8]. UVB has been shown to be highly associated with the development of squamous cell carcinomas [9]. Additionally, even suberythemal doses of UVB have been shown to induce CPD formation and therefore increased p53 expression as cells undergo apoptosis or repair [10]. UVB has also been shown to induce matrix metalloproteinases (MMPs), reactive oxygen species (ROS), and elastases involved in photoaging [11].

UVB is predominantly absorbed by the skin's epidermis, whereas UVA has a longer wavelength and therefore deeper dermal penetration, making it the primary driver of photoaging [12]. Although UVA is lower in energy than UVB, it is approximately 20 times more abundant in the earth's atmosphere and is not blocked by glass [13]. The ratio of UVB/UVA varies by season [14]. Studies of UVA on skin models demonstrated that UVA caused the induction of apoptosis in dermal fibroblasts and increased MMP levels, which are enzymes involved in collagen degradation $[12,15]$. Additionally, repeat exposure to UVA on in vivo human skin induced elevated markers of photoaging, such as ferritin and lysozyme, which are involved in the oxidative stress response and elastin degradation, respectively [16]. In a study looking at asymmetric UVA exposure of the face, chronic exposure to UVA significantly affected the clinical level of wrinkling and roughness of the skin [17]. Furthermore, in a study of 22 participants exposed to multiple sessions of low-dose UVA1, increasing levels of MMP-1 and MMP-3 were observed in a dose-dependent response in the dermis, further highlighting the role of UVA in collagen breakdown and photoaging [13]. In skin of color, UVA has been shown to induce irregular spotty pigmentation associated with photoaging [12].

However, the effects of UVA and UVB are not always distinct, as overlapping cutaneous biologic effects have been observed. UVA has been shown to induce CPDs through ROS generated by photo-activation of UVA-absorbing molecules (chromophores) in the skin such as riboflavin, porphyrins, and heme-containing proteins [18]. Similarly, UVB has also been shown to induce dermal fibroblast senescence [19].

There is increasing evidence that infrared light (IR; 700 $\mathrm{nm}-1 \mathrm{~mm})$ and VL $(400-700 \mathrm{~nm})$, predominantly in the blue light range (380-455 $\mathrm{nm}$ ), play a role in photodamage and photoaging. Studies have demonstrated that VL can independently generate ROS, proinflammatory cytokines, and MMP-1 expression and potentiate the effects of UVR [20-23]. Effects of photoaging have also been observed with irradiation of skin within the UV/VL boundary region (385-405 nm), demonstrating differential expression of genes involved in inflammation, oxidative stress, and photoaging when compared with nonirradiated skin [24]. Likewise, in vivo skin irradiated with IR and VL has shown significantly increased MMP-1 and MMP-9 expression and decreased type I procollagen expression, implicating IR and VL light in the degradation of dermal collagen [25]. Moreover, studies have demonstrated that there is a synergistic relationship between even small amounts of UVA1 and VL in the induction of increased and prolonged pigmentation $[21,26]$. This suggests that VL and IR may play a significant but underreported role in photoaging and dyspigmentation.

Although the exact mechanisms are not yet fully understood, increasing literature indicates a need for photoprotection against the broad spectrum of electromagnetic radiation (UV, VL, and IR) to prevent photoaging.

\section{Role of Sunscreens in Photoaging}

The concept of a topical photoprotective product has been around since the times of the ancient Egyptians in 4000 BC, but the first commercial sunscreens were not available until 
the 1920-1930s [27, 28]. At that time, understanding of UV radiation was limited and focused mainly on UVB protection. With the increasing popularity of sunscreen over the years, the concept of standardization of photoprotection against UVB was introduced [27]. SPF was recognized by the FDA in 1978 as the standard for measuring sun protection [27].

UV-induced erythema is mostly attributed to UVB, with a minor contribution by UVA2. The concept of SPF, an assessment using UV-induced erythema as an endpoint, as a sole measurement of sun protection persisted for many decades despite advances in the study of UVR suggesting that UVA may play a significant role in photoaging $[27,29$, 30]. In 1992, the UVA star rating system was created by The Boots Company in the UK but was not widely implemented [27]. Although other methods of evaluating the efficacy of UVA filters have been proposed, the FDA currently uses critical wavelength $(\mathrm{CW})$ determination. With this method, sunscreen products whose $90 \% \mathrm{UV}$ absorbance occurs at $\geq 370 \mathrm{~nm}$ are allowed to be labeled as "broad spectrum" [31]. In Europe, the International Organization Standardization 24443 guidelines use a minimum ratio of UVA protection factor to SPF of 1:3 for all marketed sunscreens [32]. In a study of 20 sunscreens tested against the FDA guidelines and the ISO 24443 guidelines, 19 of 20 sunscreens met the $\mathrm{CW}$ requirements set by the FDA, whereas only 11 of 20 sunscreens met the ISO 24443 standard [31]. To address this disparity, the FDA proposed a new rule on sunscreens in 2019 that specifically highlighted a requirement for a UVA1 $(340-400 \mathrm{~nm})$ to UVA and UVB $(290-400 \mathrm{~nm})$ ratio of $\geq 0.7$; however, the FDA has not yet made a final decision [33]. Clearly, there exists further need for global standardization to help protect and guide consumers.

In recent years, tinted sunscreens have become more prevalent as a means of protection against VL. Most FDAapproved compounds for UV protection do not adequately protect against VL because compounds must be opaque to filter VL [34]. Zinc oxide and titanium dioxide can protect against VL but only when they are pigmentary grade and not micronized. Tinted sunscreens incorporate combinations of iron oxides and pigmentary titanium dioxide to offer VL protection and utilize the different colors of iron oxides and pigmentary titanium dioxide to improve color match on people of all Fitzpatrick skin types [34, 35]. It should be noted that iron oxides are not considered to be UV filters so are listed under "inactive ingredients" on sunscreen product packages, whereas pigmentary-grade titanium dioxide and zinc oxide are FDA-approved inorganic filters. However, the exact efficacy of specific tinted sunscreens for VL protection has been largely unregulated as no standards or guidelines for VL protection yet exist. A method for VL protection factor has been recently suggested using in vivo assessment in melano-competent subjects $[22,36]$.
There is good evidence that daily photoprotection and daily sunscreen use plays an important role in the prevention of photoaging $[37,38]$. In a study of 46 patients randomly selected to use vehicle or sunscreens with UVA and UVB protection daily for 24 months, a significant histological difference in solar elastosis was observed in the vehicle versus treatment group [38]. Furthermore, in a study of 12 subjects in which each subject was exposed to one minimal erythemal dose of simulated solar radiation to three areas of buttock skin (unprotected skin, vehicle, and day cream with UVA and UVB protection) and control (no exposure), the unprotected skin demonstrated significant melanization, increased stratum corneum and stratum granulosum thickness, elevated expression of tenascin, reduced type I procollagen, and slightly increased lysozyme and alpha- 1 antitrypsin, which were all mitigated by the day cream-sunscreen combination [39]. Not only have sunscreens been shown to prevent photoaging but evidence also suggests that they may play a role in the reversal of extrinsic aging. In a prospective study, 32 subjects were asked to apply daily broad-spectrum photostable sunscreen (SPF 30) for 52 weeks. At the end of the study, significant improvements in skin texture, clarity, and mottled and discrete pigmentation were observed, with $100 \%$ of subjects showing improvement in skin clarity and texture [40]. However, further research into the molecular mechanism of sunscreen's effects on the reversal of chronologic aging must be performed.

\section{Challenges and Limitations of Current Sunscreens}

Sunscreen technology has made great advancements in accessibility, consumer acceptability, and overall safety and efficacy over the years. However, the challenges and limitations of current sunscreens leave room for further research and innovation. In the evaluation of sunscreens available for US consumers today, FDA regulations, safety in humans, and safety for the environment must be carefully considered.

In the 2019 proposed rule on sunscreens, the FDA proposed to categorize sunscreen filters as category I-“GRASE" (Generally Recognized as Safe and Effective), category II-non GRASE, or category III-requires further evaluation (Table 1) [41]. Currently, only two UV filters are category I: titanium dioxide and zinc oxide [42]. Both of these inorganic filters work by scattering, reflecting, and absorbing UV. The aggregation of these particles on the skin means they tend to leave a whitish hue on the skin that is unacceptable for many consumers, especially those with skin of color [43, 44].

In the 2019 FDA-proposed rule, two ingredients, paraaminobenzoic acid (PABA) and trolamine salicylate, were classified as category II and banned from products 
marketed in the USA given their safety concerns. PABA has been linked to cases of allergic and photoallergic dermatitis and is a cross-sensitizer to sulfonamide antibiotics, thiazide diuretics, local anesthetics, and dyes [42]. Trolamine salicylate is a salicylate class of UV filters and has been linked to systemic absorption and increased risk of bleeding and salicylate toxicity [42]. It should be noted that neither of these has been used in the US market for years, so this categorization does not affect the US market.

Organic UV filters, dioxybenzone, sulisobenzone, oxybenzone, avobenzone, cinoxate, octinoxate, octisalate, homosalate, padimate $\mathrm{O}$, ensulizole, meradimate, and octocrylene have now been categorized as category III, which means that additional data to determine the general recognition of safety is needed [42]. Organic UV filters absorb the higher energy of UV rays and emit a lower thermal energy $[41,45]$. It should be noted that the FDA is only requesting safety data for these 12 filters and did not question the efficacy of UV filters. None of the 12 category III UV filters offer effective visible light protection, and only meradimate and avobenzone offer partial UVA1 protection [41].

The organic UV filters can be categorized into cinnamates, benzophenones, salicylates, PABA derivatives, and others. Octinoxate, a cinnamate, is the most common sunscreen ingredient in the USA. It is photolabile and is often combined with other UVB absorbers to increase both its final SPF and its photostability [46].

The benzophenones include dioxybenzone, sulisobenzone, oxybenzone, and avobenzone, with oxybenzone the

Table 1 US FDA-approved ultraviolet filters

\begin{tabular}{|c|c|c|c|c|}
\hline Ultraviolet filters & Category $^{\mathrm{a}}$ & $\begin{array}{l}\text { Maximum } \\
\text { concentration } \\
(\%)\end{array}$ & Peak absorption (nm) & Protection against \\
\hline \multicolumn{5}{|l|}{ Inorganic filters } \\
\hline Titanium dioxide & GRASE (I) & 25 & Dependent on particle size & UVB, UVA2, UVA1, visible light \\
\hline Zinc oxide & GRASE (I) & 25 & Dependent on particle size & UVB, UVA2, UVA1, visible light \\
\hline \multicolumn{5}{|l|}{ Organic filters } \\
\hline \multicolumn{5}{|l|}{ Benzophenones } \\
\hline Dioxybenzone (benzophenone-8) & Non GRASE (III) & 3 & 352 & UVB, UVA2 \\
\hline Oxybenzone (benzophenone-3) & Non GRASE (III) & 6 & 288,325 & UVB, UVA2 \\
\hline Sulisobenzone (benzophenone-4) & Non GRASE (III) & 10 & 366 & UVB, UVA2 \\
\hline \multicolumn{5}{|l|}{ Cinnamates } \\
\hline Cinoxate & Non GRASE (III) & 3 & 289 & UVB \\
\hline $\begin{array}{l}\text { Octinoxate (octyl methoxycinnamate, } \\
\text { Parsol MCX) }\end{array}$ & Non GRASE (III) & 8 & 311 & UVB, UVA2 \\
\hline \multicolumn{5}{|l|}{ Others } \\
\hline $\begin{array}{l}\text { Butyl methoxydibenzoyl methane } \\
\text { (avobenzone, Parsol 1789) }\end{array}$ & Non GRASE (III) & 3 & 360 & UVA1 \\
\hline $\begin{array}{l}\text { Ecamsule (terephthalylidene dicamphor } \\
\text { sulfonic acid) }\end{array}$ & No GRASE rating ${ }^{\mathrm{b}}$ & 3 & NA & UVA1, UVA2 \\
\hline $\begin{array}{l}\text { Ensulizole (phenylbenzimidazole } \\
\text { sulfonic acid) }\end{array}$ & Non GRASE (III) & 4 & 310 & UVB, UVA2 \\
\hline Meradimate (menthyl anthranilate) & Non GRASE (III) & 5 & 340 & UVA1, UVA2 \\
\hline Octocrylene & Non GRASE (III) & 10 & 303 & UVB, UVA2 \\
\hline \multicolumn{5}{|l|}{ PABA derivatives } \\
\hline Padimate $\mathrm{O}$ (octyl dimethyl PABA) & Non GRASE (III) & 8 & 311 & UVB \\
\hline Para-aminobenzoic acid (PABA) & Non GRASE (II) & 15 & 283 & UVB \\
\hline \multicolumn{5}{|l|}{ Salicylates } \\
\hline Homosalate (homomethyl salicylate) & Non GRASE (III) & 15 & 306 & UVB, UVA2 \\
\hline Octisalate (octyl salicylate) & Non GRASE (III) & 5 & 307 & UVB, UVA2 \\
\hline Trolamine salicylate (TEA salicylate) & Non GRASE (II) & 12 & $260-355$ & UVB \\
\hline
\end{tabular}

GRASE generally recognized as safe and effective, $N A$ not applicable, $P A B A$ para-aminobenzoic acid, $U V$ ultraviolet

a2019 FDA Proposed Rule has suggested three categories: I-GRASE; II—non GRASE; III—insufficient safety data to make a positive GRASE determination

${ }^{\mathrm{b}}$ Approved through new drug application process 
most commonly used agent in the group [46]. Although benzophenones have been shown to be effective UVA filters, their lack of photostability requires them to be compounded with other filters such as octocrylene, salicylates, micronized zinc oxide, and titanium dioxide to improve their photostability [44, 46, 47]. Additionally, oxybenzone is the most common photoallergen of the UV filters.

The salicylates octisalate and homosalate are only weak UVB absorbers and are mainly used in sunscreens as photostabilizers in combination with other organic filters [46]. Padimate $\mathrm{O}$ is a PABA derivative; like its predecessor, it has potent UVB filtration but is rarely used [44, 46]. Ensulizole is primarily a UVB filter with minimal UVA2 activity [48]. Meradimate is a weak UVA blocker and has no activity against UVB [41, 46]. Octocrylene is a photostable UVB and UVA2 filter primarily used as a photostabilizer in conjunction with other filters [46]. Ecamsule (Mexoryl SX) is an effective UVA filter that has been shown to be effective against photoaging when combined with UVB filters [49]. It has been approved via the new drug application process, with its use as an active ingredient permitted only in certain products under specific concentrations [41, 43, 44].

Although other photostable and more effective broadspectrum UV filters, including bemotrizinol, bisoctrizole, and drometrizole trisiloxane, are available in other countries, these agents - along with many other UV filters available in other countries-are still pending FDA approval in the USA [27, 41]. In over a decade, no new UV filters have been approved by the FDA to be added to the 16 currently approved filters. In contrast, the European Commission currently has 27 approved UV filters [27]. However, with the Coronavirus Aid, Relief, and Economic Security (CARES) Act signed into law in March 2020, the FDA has been mandated to move from a laborious rulemaking process to an administrative order process, which means it should not take as long to implement a monograph. The FDA is to issue a new proposed administrative order by 27 September 2021. Once the final administrative order has been enacted, industry has 12 months to comply. In addition, the CARES Act also incentivizes innovation by providing an 18-month exclusivity period to the requesting manufacturer of a new filter [50].

Controversy regarding organic sunscreen safety in humans has increasingly been a topic of discussion after studies showed systemic absorption of six commonly used sunscreen active ingredients [51, 52]. This 2020 study of 48 randomized participants applying $2 \mathrm{mg} /$ $\mathrm{cm}^{2}$ of sunscreen product to $75 \%$ of body surface areas between one and four times per day for 4 days demonstrated systemic absorption of avobenzone, oxybenzone, octocrylene, homosalate, octisalate, and octinoxate [51]. However, a systematic review of 29 studies looking at the effects of two of the most commonly studied sunscreen ingredients-oxybenzone and octinoxate-demonstrated that oxybenzone had no adverse effects on male and female fertility, female reproductive hormone levels, adiposity, fetal growth, childhood neurodevelopment, or sexual maturation, and octinoxate had no effect on thyroid and reproductive hormone levels [53]. Although the review recommended further research into the effects of oxybenzone levels on thyroid hormone, testosterone level, kidney function, and pubertal timing, the evidence is not yet sufficient to support a causal relationship between the elevated systemic levels of oxybenzone or octinoxate and adverse health outcomes. Further longitudinal randomized controlled studies should be performed before factoring the biological effects of systemically absorbed agents into clinical and practical guidelines [54, 55]. A recent report by Valisure LLC, an independent laboratory, also raised safety concerns regarding benzene in sunscreen products. After testing multiple batches of 69 brands of sunscreen and after-sun skincare products, they found that 78 batches contained elevated levels of benzene, a carcinogen known to cause leukemia and lymphoma [56]. It is important to note that both organic and inorganic sunscreens and some cosmetic products that did not contain any UV filters were among the contaminated products. In addition, many sunscreen products tested did not contain benzene. The report concluded that the contamination was due to supply chain issues in the manufacturing process rather than degradation of sunscreen filters. These findings led to an FDA citizen petition for the recall of identified batches of sunscreen with elevated levels of benzene and further investigation into these products and their manufacturing processes. A full report, including a list of products tested, can be found on the Valisure website [57].

Additionally, the National Oceanic and Atmospheric Administration identified ten sunscreen ingredients as being toxic to coral and marine life: oxybenzone, benzophenone-1, benzophenone-8, PABA, 4-methylbenzylidene camphor, 3-benzylidene camphor, nano-titanium dioxide, nano-zinc oxide, octinoxate, and octocrylene [58]. Studies that demonstrated marine toxicity were performed in vitro with high concentrations of sunscreen ingredients $[44,55$, 59]. In a review looking at all 32 published studies until June 2020, 14 different organic UV filters in seawater near coral reefs were detected in the nanograms per liter range, in contrast to toxic levels in the micrograms per liter to milligrams per liter range reported in nine papers [60]. This puts the toxic levels of organic UV filters at 1000to 1 million-fold higher concentrations than currently reported. Although 27 of the 32 reviewed studies showed no risk of UV filters to coral reefs, three studies of oxybenzone and octinoxate demonstrated a few data points where some risk was present [54]. This reflects the major data 
gaps that immediately need to be addressed with highquality monitoring and toxicity studies applicable to the real world. To address this issue, on 9 February 2021, the National Academies formed a committee sponsored by the Environmental Protection Agency to study the environmental and health impacts of sunscreens. Although data supporting that the coral reefs are adversely impacted by environmental exposure to UV filters are limited, the state of Hawaii banned sunscreens containing oxybenzone and octinoxate in 2018, and Key West, Florida, USA, did the same in 2019 [59].

Although FDA guidelines aim to protect US consumers from harm, it has also greatly diminished the variety of UV filters available to consumers. Newer and more effective broad-spectrum UV filters are available in other countries but are not currently FDA approved [41]. With the new proposed administrative order under the CARES Act and careful consideration of human safety, environmental safety, photostability, and consumer cosmesis, the development and approval of new sunscreens that are effective against UVA, UVB, and VL must be considered for protection against photoaging.

\section{Additives in Sunscreens}

With the rise of cosmeceuticals and additives in sunscreens, it is important to evaluate the safety and efficacy of these substances. Although the exact mechanism of UVR- and VL-induced photoaging is still being explored, the downstream effects of increased ROS, MMPs, and DNA damage have been widely reported $[8,11]$. To combat the deleterious effects of sunlight on the skin, additives have been used or proposed in sunscreens to enhance photoprotection and help prevent photoaging.

Antioxidants play an important role in preventing, ameliorating, and dampening free radicals and oxidative stress. Although our bodies produce natural antioxidants, UVR and other stressors can often overwhelm our endogenous supply [61]. Topical antioxidants have been formulated into sunscreens to replenish depleted antioxidant supplies and diminish oxidative stress on the skin. Yet the exact role and efficacy of antioxidants in sunscreens remains controversial. A 2011 ex vivo study by Wang et al. [62] evaluated the radical skin protection factor (RSF) and antioxidant power (AP) of 12 sunscreen products containing vitamin $\mathrm{C}$, vitamin $\mathrm{E}$, or other antioxidant substances against simulated UVA- and UVB-induced ROS. RSF was defined as the ratio of free radicals in unprotected skin to protected skin, and AP evaluates the capacity and reaction time of antioxidants by measuring free electron spin [62]. They demonstrated that the RSF correlated with the UVA RSF rather than any antioxidant ingredients [62]. However, the study was performed ex vivo and may not correlate to in vivo responses in humans. More recent reviews and studies have demonstrated positive effects of the addition of antioxidants into sunscreen formulations. For example, a study looking at skin irradiated with UVB found that sunscreens with SPF 25 and a mixture of caffeine, vitamin E, vitamin C, Echinacea pallida extract, gorgonian extract, and chamomile essential oil demonstrated less MMP-1 expression than those with only SPF 25 [63]. The variability in the efficacy of antioxidants in sunscreens may depend on the formulation of the sunscreen. It has been proposed that, for antioxidants to be efficacious, they must have high antioxidative capacities, be present in high concentrations, be stable in the final formulation, and be able to penetrate the stratum corneum and still exist at high enough concentrations in the epidermis and dermis to be effective [61].

In terms of antioxidants that have been explored in topical formulations, vitamin $\mathrm{C}$ ( $\mathrm{L}$-ascorbic acid) is the predominant antioxidant in the skin and plays an important role in the skin's aqueous compartments because of its water solubility [61]. It also helps replenish vitamin E, acts as a cofactor in collagen synthesis, and reduces elastin accumulation [61]. It is not synthesized by the human body and must be replenished via oral intake [64]. Additionally, because of its ionic charge at physiologic $\mathrm{pH}$, it cannot penetrate the stratum corneum without becoming unstable. Fortunately, a stable formulation can be made by compounding it with other antioxidants: vitamin E (alpha-tocopherol) and ferulic acid [61, 64]. Murray et al. [65] demonstrated that skin irradiated with solarsimulated UVR after application of a topical formulation of 15\% L-ascorbic acid, 1\% alpha-tocopherol, and 0.5\% ferulic acid (CEFer) for 4 days significantly decreased UVinduced erythema, sunburn cells, thymine dimers, and p53 induction when compared with untreated skin. Furthermore, vitamin $\mathrm{E}$ has been shown to be effective in the reduction of lipid peroxidation, photoaging, immunosuppression, and photocarcinogenesis in multiple animal and human studies [61]. This suggests a role for topical CEFer in protecting against photoaging and skin cancers $[64,65]$.

Vitamin A and its derivatives, mainly retinoids and carotenoids, have been well studied in the realm of antiaging and have shown benefit in the prevention and reversal of photoaging [66]. They bind to cytoplasmic receptors such as cellular retinoic acid-binding protein types I and II and cellular retinol-binding protein as well as nuclear receptors such as nuclear retinoic acid receptors and retinoid X receptors to inhibit activation of protein-1 and MMP-1 expression [61]. This leads to increased epidermal proliferation, leading to epidermal thickening, compaction of the stratum corneum, synthesis and deposition of glycosaminoglycans, and increased collagen production $[61,67]$. Furthermore, there 
is evidence that topical retinoids may play a role in chemoprevention of nonmelanoma skin cancers through initiating growth arrest of tumor cells and normal cellular differentiation [68]. However, given the relative instability of retinol and retinoids when exposed to UV and visible light, their use as a sunscreen additive is predominantly for their anti-aging effects and not for increased photoprotection. They are rarely found in recreational sunscreens, and their stability is highly dependent on their formulation and chemical structure. For example, when tretinoin is compounded in ethanol, it undergoes isomerization within just a few seconds when irradiated with light of 300-800 nm [69]. The stability of tretinoin is improved when incorporated into liposomes [69]. Retinyl palmitate is an ester of retinol that is widely used in cosmetic products because of their high thermal stability when compared with retinol [70]. A study of 11 healthy volunteers using two formulations of retinyl palmitate for 60 days reported significant improvements in skin smoothness, skin roughness, scaliness, and wrinkles with both formulations [71]. Retinyl palmitate can be compounded with photostabilizers and UV filters and loaded onto nanotechnology-based drug-delivery systems to improve stability and drug penetration, but large-scale randomized controlled trials are needed to study the antiaging properties of these formulations [70, 72]. Additionally, concerns have been raised regarding an increase in cutaneous malignancy with simultaneous use of topical retinyl palmitate and UVR exposure. A recent study looking at SKH-1 hairless mice treated with control cream or creams containing retinyl palmitate and subsequently irradiated with simulated solar light demonstrated an increased risk of photo-co-carcinogenesis in the group using cream containing retinyl palmitate [73]. However, these claims have not been largely substantiated or reported in humans and need to be further studied.

Other antioxidants that have been reported in the literature include soy extracts, polyphenols, melatonin, algae extract, and Polypodium leucotomos extract [30]. A study of 68 participants observed that soy moisturizer containing soybean-derived serine protease inhibitors (soybean trypsin inhibitor and Bowman-Birk protease inhibitor) significantly improved mottled pigmentation, blotchiness, dullness, fine lines, overall texture, overall skin tone, and overall appearance when compared with vehicle [74]. This positive clinical effect may be related to the role of soybean-derived serine protease inhibitors on the regulation of keratinocytes through keratinocyte protease-activated receptor 2 , but additional studies must be performed to further elucidate its mechanism [74].

Polyphenols are found in many botanicals, including tea leaves, grape seeds (Vitis vinifera), blueberries, almond seeds, and pomegranate extract [75]. In a study of five participants, sunscreen compounded with tea extracts containing polyphenols such as epigallocatechin-3-gallate better protected human skin against solar-simulated UVR over sunscreen alone in regards to decreasing MMP-1 [63]. Additionally, green tea extract compounded with resveratrol, another polyphenol, provided SPF protection independent of physical and chemical UV filters, but additional in vivo studies must be performed to fully assess its effectiveness [76].

Melatonin acts as an antioxidant in three different but complementary ways. It can act as a free radical scavenger, decrease free radical generation, and upregulate antioxidant enzymes [77]. It has shown promise against both UVB- and UVA-induced oxidative stress. In studies of human melanocytes and keratinocytes, cells pretreated with melatonin decreased p53 expression, improved DNA repair, and decreased CPD generation [78, 79]. An in vitro study of mouse fibroblast cells (NIH3T3) pretreated with melatonin and irradiated with UVA demonstrated increased heme-degrading enzymes and suppression of UVA-induced photodamage when compared with untreated irradiated cells [77]. Additionally, melatonin protected against UV-induced erythema and activated endogenous enzymes to act against oxidative stress [75]. This suggests a potential role of melatonin as an additive to protect keratinocytes, melanocytes, and fibroblasts against UV-induced photoaging.

Many studies have shown that multicellular algae not only have UV-absorbing properties but also provide benefits against oxidative stress [75]. Mycosporine-like amino acids (MAAs) produced by algae are potent UV filters with maximum absorption between 310 and $362 \mathrm{~nm}$ [80]. Shinorine is a commercialized MAA extracted from a type of red algae, Porphyra umbilicalis, and has already been used in sunscreens produced by two European companies [81]. Furthermore, the algae and algae products have also demonstrated protective properties against photoaging. Alga Corallina pilulifera methanol extract reduced MMP-2 and MMP-9 in UV-irradiated human dermal fibroblasts [82]. Additionally, many species of brown algae are protective against photo-oxidative stress [75]. With controversies around chemical sunscreens and their effects on marine life, algae-derived sunscreens may provide a future solution for eco-friendly photoprotection; however, most formulations of sunscreens with MAAs currently contain only a very small percentage of this active ingredient, and it functions as an adjuvant to UV filters and other sources of photoprotection [83].

Polypodium leucotomos extract (PLE) is derived from a tropical fern found in Central and South America and has antioxidative, chemoprotective, immunomodulatory, and anti-inflammatory effects $[84,85]$. In a recent study of 22 individuals irradiated with UVB, UVA, and VL, oral PLE demonstrated suppressive effects on UVB-induced erythema within $2 \mathrm{~h}$ of administration [84]. Oral PLE demonstrated similar photoprotective effects against VL. In a cross-over study, subjects taking PLE $480 \mathrm{mg}$ daily 
demonstrated a significant decrease in persistent pigment darkening, delayed tanning, and cyclooxygenase- 2 compared with pre-PLE $[86,87]$. Oral PLE should be taken daily to receive benefit and is meant to be an adjuvant to sunscreen, not a replacement. Topical formulations of PLE were also effective in reducing sunburn cells and reducing CPD in an in vitro reconstructed human epidermis model [87]. However, future in vivo studies must be performed to better assess the feasibility of topical PLE as a sunscreen additive.

In addition to antioxidants, photolyases are also beneficial additives in sunscreens. Photolyases are enzymes with a unique ability to repair DNA damage, specifically CPDs. They are flavoproteins and require flavonoids as cofactors to absorb UV radiation. The absorbed energy from UV radiation is then transferred to damaged DNA to break CPD bonds in both in vivo and in vitro studies [30]. It also significantly reduced markers of photoaging when added to SPF 50 sunscreen and antioxidants compared with sunscreen alone or sunscreen and antioxidants [88]. This suggests that photolyases may synergistically enhance the photoprotective effects of sunscreens and antioxidants [30].

\section{Summary}

The perception of sunscreen use has shifted from purely protecting against $\mathrm{UV}$-induced erythema to broad-spectrum protection against not only erythema but also photoaging, dyspigmentation, DNA damage, and photocarcinogenesis. The impact of visible light and IR light in photoaging is still being explored, but better methods of protection against these wavelengths are needed. Sunscreens continue to be adapted to provide the broadest coverage while being cosmetically appealing. However, with the increased scrutiny of UV filters in the 2019 FDA proposed rule, new UV filters that are safe for humans and the environment, photostable, and consumer friendly must be developed and approved to offer continued sun protection for US consumers. When choosing a sunscreen, a broad-spectrum tinted sunscreen with SPF $\geq 30$ used daily will offer protection against UVR and VL to reduce their effects on photoaging. Additionally, sunscreen additives such as antioxidants, photolyases, and more have opened the door for not only improved photoprotection against but also the reversal of skin aging. However, larger-scale and replicable studies must be performed before clinical guidelines can be issued.

\section{Declarations}

Funding No sources of funding were used to conduct this study or prepare this manuscript.
Conflict of interest LG has no conflicts of interest that are directly relevant to the content of this article. HWL has served as coinvestigator for studies sponsored by Incyte, L'Oreal, Pfizer, and PCOI; as consultant for Pierre Fabre, ISDIN, Ferndale, La Roche-Posay, and Beiersdorf; and as a speaker on general educational sessions for La Roche-Posay and Cantabria Labs. TFM has served as subinvestigator for Allergan and Ferndale Laboratories.

Availability of data and material Not applicable.

Ethics approval Not applicable.

Consent Not applicable.

Author contributions All authors were significant contributors to the concept and planning, drafting, and revision of the manuscript. All authors approved the final submitted version of the manuscript.

\section{References}

1. Yaar M, Gilchrest BA. Photoageing: mechanism, prevention and therapy. Br J Dermatol. 2007;157:874-87.

2. Sachs DL, Varani J, Chubb H, Fligiel SEG, Cui Y, Calderone K, et al. Atrophic and hypertrophic photoaging: clinical, histologic, and molecular features of 2 distinct phenotypes of photoaged skin. J Am Acad Dermatol. 2019;81:480-8.

3. Anti-Aging Products-Market Study by Global Industry Analysts, Inc. [Internet]. [cited 2021 Mar 10]. Available from: https:// www.strategyr.com/market-report-anti-aging-products-forec asts-global-industry-analysts-inc.asp.

4. Poon F, Kang S, Chien AL. Mechanisms and treatments of photoaging. Photodermatol Photoimmunol Photomed. 2015;31:65-74.

5. Flament F, Bazin R, Laquieze S, Rubert V, Simonpietri E, Piot B. Effect of the sun on visible clinical signs of aging in Caucasian skin. Clin Cosmet Investig Dermatol. 2013;6:221-32.

6. Young AR, Claveau J, Rossi AB. Ultraviolet radiation and the skin: photobiology and sunscreen photoprotection. J Am Acad Dermatol. 2017;76:S100-9.

7. Miyamura Y, Coelho SG, Schlenz K, Batzer J, Smuda C, Choi $\mathrm{W}$, et al. The deceptive nature of UVA-tanning versus the modest protective effects of UVB-tanning on human skin. Pigment Cell Melanoma Res. 2011;24:136-47.

8. Marrot L, Meunier J-R. Skin DNA photodamage and its biological consequences. J Am Acad Dermatol (Elsevier). 2008;58:S139-48.

9. de Gruijl FR, Sterenborg HJ, Forbes PD, Davies RE, Cole C, Kelfkens G, et al. Wavelength dependence of skin cancer induction by ultraviolet irradiation of albino hairless mice. Cancer Res. 1993;53:53-60.

10. Seité S, Fourtanier A, Moyal D, Young AR. Photodamage to human skin by suberythemal exposure to solar ultraviolet radiation can be attenuated by sunscreens: a review. Br J Dermatol. 2010;163:903-14.

11. Pillai S, Oresajo C, Hayward J. Ultraviolet radiation and skin aging: roles of reactive oxygen species, inflammation and protease activation, and strategies for prevention of inflammation-induced matrix degradation-a review. Int J Cosmet Sci. 2005;27:17-34.

12. Battie C, Jitsukawa S, Bernerd F, Bino SD, Marionnet C, Verschoore M. New insights in photoaging, UVA induced damage and skin types. Exp Dermatol. 2014;23:7-12.

13. Wang F, Smith NR, Tran BAP, Kang S, Voorhees JJ, Fisher GJ. Dermal damage promoted by repeated low-level UV-A1 
exposure despite tanning response in human skin. JAMA Dermatol. 2014;150:401.

14. Cole C, VanFossen R. Measurement of sunscreen UVA protection: an unsensitized human model. J Am Acad Dermatol. 1992;26:178-84.

15. Lee YK, Cha HJ, Hong M, Yoon Y, Lee H, An S. Role of NF- $\kappa$ B-p53 crosstalk in ultraviolet A-induced cell death and G1 arrest in human dermal fibroblasts. Arch Dermatol Res. 2012;304:73-9.

16. Séite S, Moyal D, Richard S, de Rigal J, Lévêque JL, Hourseau C, et al. Mexoryl SX: a broad absorption UVA filter protects human skin from the effects of repeated suberythemal doses of UVA. J Photochem Photobiol B. 1998;44:69-76.

17. Mac-Mary S, Sainthillier J-M, Jeudy A, Sladen C, Williams C, Bell M, et al. Assessment of cumulative exposure to UVA through the study of asymmetrical facial skin aging. Clin Interv Aging. 2010;5:277-84.

18. McMillan TJ, Leatherman E, Ridley A, Shorrocks J, Tobi SE, Whiteside JR. Cellular effects of long wavelength UV light (UVA) in mammalian cells. J Pharm Pharmacol. 2008;60:969-76.

19. Cavinato M, Jansen-Dürr P. Molecular mechanisms of UVBinduced senescence of dermal fibroblasts and its relevance for photoaging of the human skin. Exp Gerontol. 2017;94:78-82.

20. Liebel F, Kaur S, Ruvolo E, Kollias N, Southall MD. Irradiation of skin with visible light induces reactive oxygen species and matrix-degrading enzymes. J Invest Dermatol. 2012;132:1901-7.

21. Kohli I, Chaowattanapanit S, Mohammad TF, Nicholson CL, Fatima S, Jacobsen G, et al. Synergistic effects of long-wavelength ultraviolet al and visible light on pigmentation and erythema. $\mathrm{Br}$ J Dermatol. 2018;178:1173-80.

22. Kohli I, Nahhas AF, Braunberger TL, Chaowattanapanit S, Mohammad TF, Nicholson CL, et al. Spectral characteristics of visible light-induced pigmentation and visible light protection factor. Photodermatol Photoimmunol Photomed. 2019;35:393-9.

23. Mahmoud BH, Hexsel CL, Hamzavi IH, Lim HW. Effects of visible light on the skin. Photochem Photobiol. 2008;84:450-62.

24. Lawrence KP, Douki T, Sarkany RPE, Acker S, Herzog B, Young AR. The UV/Visible Radiation Boundary Region (385-405 nm) damages skin cells and induces "dark" cyclobutane pyrimidine dimers in human skin in vivo. Sci Rep. 2018;8:12722.

25. Cho S, Lee MJ, Kim MS, Lee S, Kim YK, Lee DH, et al. Infrared plus visible light and heat from natural sunlight participate in the expression of MMPs and type I procollagen as well as infiltration of inflammatory cell in human skin in vivo. J Dermatol Sci. 2008;50:123-33.

26. Ruvolo E, Fair M, Hutson A, Liebel F. Photoprotection against visible light-induced pigmentation. Int J Cosmet Sci. 2018;40:589-95.

27. Ma Y, Yoo J. History of sunscreen: an updated view. J Cosmet Dermatol. 2021;20:1044-9.

28. Aldahan AS, Shah VV, Mlacker S, Nouri K. The history of sunscreen. JAMA Dermatol. 2015;151:1316.

29. Kligman AM. Early destructive effect of sunlight on human skin. JAMA. 1969;210:2377-80.

30. Yeager DG, Lim HW. What's new in photoprotection: a review of new concepts and controversies. Dermatol Clin. 2019;37:149-57.

31. Wang SQ, Xu H, Stanfield JW, Osterwalder U, Herzog B. Comparison of ultraviolet A light protection standards in the United States and European Union through in vitro measurements of commercially available sunscreens. J Am Acad Dermatol (Elsevier). 2017;77:42-7.

32. 14:00-17:00. ISO 24443:2012 [Internet]. ISO. [cited $2021 \mathrm{Mar}$ 21]. Available from: https://www.iso.org/cms/render/live/en/sites/ isoorg/contents/data/standard/04/65/46522.html.
33. Wang SQ, Lim HW. Highlights and implications of the 2019 proposed rule on sunscreens by the US Food and Drug Administration. J Am Acad Dermatol. 2019;81:650-1.

34. Lyons AB, Trullas C, Kohli I, Hamzavi IH, Lim HW. Photoprotection beyond ultraviolet radiation: a review of tinted sunscreens. J Am Acad Dermatol. 2021;84:1393-7.

35. Geisler AN, Austin E, Nguyen J, Hamzavi I, Jagdeo J, Lim HW. Visible light Part II. Photoprotection against visible and ultraviolet light. J Am Acad Dermatol. 2021;84:1233-44.

36. Lim HW, Kohli I, Granger C, Trullàs C, Piquero-Casals J, Narda $\mathrm{M}$, et al. Photoprotection of the skin from visible light-induced Pigmentation: current testing methods and proposed harmonization. J Invest Dermatol. 2021;S0022-202X(21)01123-4.

37. Hughes MCB, Williams GM, Baker P, Green AC. Sunscreen and prevention of skin aging: a randomized trial. Ann Intern Med. 2013;158:781-90.

38. Boyd AS, Naylor M, Cameron GS, Pearse AD, Gaskell SA, Neldner KH. The effects of chronic sunscreen use on the histologic changes of dermatoheliosis. J Am Acad Dermatol. 1995;33:941-6.

39. Seité S, Fourtanier AMA. The benefit of daily photoprotection. J Am Acad Dermatol. 2008;58:S160-166.

40. Randhawa M, Wang S, Leyden JJ, Cula GO, Pagnoni A, Southall MD. Daily use of a facial broad spectrum sunscreen over one-year significantly improves clinical evaluation of photoaging. Dermatol Surg Off Publ Am Soc Dermatol Surg Al. 2016;42:1354-61.

41. Sabzevari N, Qiblawi S, Norton SA, Fivenson D. Sunscreens: UV filters to protect us: Part 1: changing regulations and choices for optimal sun protection. Int J Womens Dermatol. 2021;7:28-44.

42. Sunscreen Drug Products for Over-the-Counter Human Use [Internet]. Fed. Regist. 2019 [cited 2021 Mar 22]. Available from: https://www.federalregister.gov/documents/2019/02/26/201903019/sunscreen-drug-products-for-over-the-counter-human-use.

43. Forestier S. Rationale for sunscreen development. J Am Acad Dermatol (Elsevier). 2008;58:S133-8.

44. Mancuso JB, Maruthi R, Wang SQ, Lim HW. Sunscreens: an update. Am J Clin Dermatol. 2017;18:643-50.

45. Gabros S, Nessel TA, Zito PM. Sunscreens and photoprotection. StatPearls [Internet]. Treasure Island: StatPearls Publishing; 2021 [cited 2021 Mar 27]. Available from: http://www.ncbi. nlm.nih.gov/books/NBK537164/.

46. Kullavanijaya P, Lim HW. Photoprotection. J Am Acad Dermatol. 2005;52:937-58.

47. Kim EJ, Kim MJ, Im NR, Park SN. Photolysis of the organic UV filter, avobenzone, combined with octyl methoxycinnamate by nano-TiO2 composites. J Photochem Photobiol B. 2015;149:196-203.

48. Nedorost S. Ensulizole (phenylbenzimidazole-5-sulfonic acid) as a cause of facial dermatitis: two cases. Dermatitis. 2005; $16: 148$.

49. Seité S, Colige A, Piquemal-Vivenot P, Montastier C, Fourtanier A, Lapière C, et al. A full-UV spectrum absorbing daily use cream protects human skin against biological changes occurring in photoaging. Photodermatol Photoimmunol Photomed. 2000;16:147-55.

50. Mohammad TF, Lim HW. The important role of dermatologists in public education on sunscreens. JAMA Dermatol. 2021;157:509.

51. Matta MK, Florian J, Zusterzeel R, Pilli NR, Patel V, Volpe DA, et al. Effect of sunscreen application on plasma concentration of sunscreen active ingredients: a randomized clinical trial. JAMA. 2020;323:256.

52. Matta MK, Zusterzeel R, Pilli NR, Patel V, Volpe DA, Florian J, et al. Effect of sunscreen application under maximal use conditions on plasma concentration of sunscreen active ingredients: a randomized clinical trial. JAMA. 2019;321:2082. 
53. Suh S, Pham C, Smith J, Mesinkovska NA. The banned sunscreen ingredients and their impact on human health: a systematic review. Int J Dermatol. 2020;59:1033-42.

54. Abbasi J. FDA trials find sunscreen ingredients in blood, but risk is uncertain. JAMA. 2020;323:1431-2.

55. Fivenson D, Sabzevari N, Qiblawi S, Blitz J, Norton BB, Norton SA. Sunscreens: UV filters to protect us: Part 2-increasing awareness of UV filters and their potential toxicities to us and our environment. Int J Womens Dermatol. 2021;7:45-69.

56. Valisure Detects Benzene in Sunscreen [Internet]. Valisure. 2021 [cited 2021 Jul 6]. Available from: https://www.valisure.com/blog/ valisure-news/valisure-detects-benzene-in-sunscreen/.

57. Valisure. Re: Valisure Citizen Petition on Benzene in Sunscreen and After-sun Care Products [Internet]. [cited 2021 Jul 26]. Available from: https://www.valisure.com/wp-content/uploads/Valis ure-Citizen-Petition-on-Benzene-in-Sunscreen-and-After-sunCare-Products-v9.7.pdf.

58. US Department of Commerce NO and AA. Sunscreen chemicals and marine life [Internet]. [cited 2021 Mar 27]. Available from: https://oceanservice.noaa.gov/news/sunscreen-corals.html.

59. Tsatalis J, Burroway B, Bray F. Evaluation of "reef safe" sunscreens: labeling and cost implications for consumers. J Am Acad Dermatol (Elsevier). 2020;82:1015-7.

60. Mitchelmore CL, Burns EE, Conway A, Heyes A, Davies IA. A critical review of organic ultraviolet filter exposure, hazard, and risk to corals. Environ Toxicol Chem. 2021;40:967-88.

61. Chen L, Hu JY, Wang SQ. The role of antioxidants in photoprotection: a critical review. J Am Acad Dermatol. 2012;67:1013-24.

62. Wang SQ, Osterwalder U, Jung K. Ex vivo evaluation of radical sun protection factor in popular sunscreens with antioxidants. J Am Acad Dermatol. 2011;65:525-30.

63. Matsui MS, Hsia A, Miller JD, Hanneman K, Scull H, Cooper KD, et al. Non-sunscreen photoprotection: antioxidants add value to a sunscreen. J Investig Dermatol Symp Proc. 2009;14:56-9.

64. Lin F-H, Lin J-Y, Gupta RD, Tournas JA, Burch JA, Selim MA, et al. Ferulic acid stabilizes a solution of vitamins $\mathrm{C}$ and $\mathrm{E}$ and doubles its photoprotection of skin. J Invest Dermatol (Elsevier). 2005; 125:826-32.

65. Murray JC, Burch JA, Streilein RD, Iannacchione MA, Hall RP, Pinnell SR. A topical antioxidant solution containing vitamins $\mathrm{C}$ and $\mathrm{E}$ stabilized by ferulic acid provides protection for human skin against damage caused by ultraviolet irradiation. J Am Acad Dermatol. 2008;59:418-25.

66. Kligman AM, Grove GL, Hirose R, Leyden JJ. Topical tretinoin for photoaged skin. J Am Acad Dermatol (Elsevier). $1986 ; 15: 836-59$

67. Mukherjee S, Date A, Patravale V, Korting HC, Roeder A, Weindl G. Retinoids in the treatment of skin aging: an overview of clinical efficacy and safety. Clin Interv Aging. 2006;1:327-48.

68. Rosenthal A, Stoddard M, Chipps L, Herrmann J. Skin cancer prevention: a review of current topical options complementary to sunscreens. J Eur Acad Dermatol Venereol. 2019;33:1261-7.

69. Kryczyk-Poprawa A, Kwiecień A, Opoka W. Photostability of topical agents applied to the skin: a review. Pharmaceutics. 2019;12:10.

70. Benevenuto CG, Matteo MASD, Campos PMBGM, Gaspar LR. Influence of the photostabilizer in the photoprotective effects of a formulation containing UV-filters and vitamin A. Photochem Photobiol. 2010;86:1390-6.

71. Farooq U, Mahmood T, Shahzad Y, Yousaf AM, Akhtar N. Comparative efficacy of two anti-aging products containing retinyl palmitate in healthy human volunteers. J Cosmet Dermatol. 2018;17:454-60.
72. Oliveira MB, do Prado AH, Bernegossi J, Sato CS, Lourenço Brunetti I, Scarpa MV, et al. Topical application of retinyl palmitate-loaded nanotechnology-based drug delivery systems for the treatment of skin aging. BioMed Res Int. 2014;2014:632570.

73. Boudreau MD, Beland FA, Felton RP, Fu PP, Howard PC, Mellick PW, et al. Photo-co-carcinogenesis of topically applied retinyl palmitate in SKH-1 hairless mice. Photochem Photobiol. 2017;93:1096-114.

74. Wallo W, Nebus J, Leyden JJ. Efficacy of a soy moisturizer in photoaging: a double-blind, vehicle-controlled, 12-week study. J Drugs Dermatol JDD. 2007;6:917-22.

75. Dunaway S, Odin R, Zhou L, Ji L, Zhang Y, Kadekaro AL. Natural antioxidants: multiple mechanisms to protect skin from solar radiation. Front Pharmacol. 2018;9:392.

76. Bhattacharya S, Sherje AP. Development of resveratrol and green tea sunscreen formulation for combined photoprotective and antioxidant properties. J Drug Deliv Sci Technol. 2020;60:102000.

77. Rezzani R, Rodella LF, Favero G, Damiani G, Paganelli C, Reiter RJ. Attenuation of ultraviolet A-induced alterations in NIH3T3 dermal fibroblasts by melatonin. Br J Dermatol. 2014;170:382-91.

78. Janjetovic Z, Jarrett SG, Lee EF, Duprey C, Reiter RJ, Slominski AT. Melatonin and its metabolites protect human melanocytes against UVB-induced damage: Involvement of NRF2-mediated pathways. Sci Rep. 2017;7:1274.

79. Janjetovic Z, Nahmias ZP, Hanna S, Jarrett SG, Kim T-K, Reiter RJ, et al. Melatonin and its metabolites ameliorate ultraviolet B-induced damage in human epidermal keratinocytes. J Pineal Res. 2014;57:90-102.

80. Yang G, Cozad MA, Holland DA, Zhang Y, Luesch H, Ding Y. Photosynthetic production of sunscreen shinorine using an engineered cyanobacterium. ACS Synth Biol. 2018;7:664-71.

81. Pandika M. Looking to nature for new sunscreens. ACS Cent Sci. 2018;4:788-90.

82. Ryu B, Qian Z-J, Kim M-M, Nam KW, Kim S-K. Anti-photoaging activity and inhibition of matrix metalloproteinase (MMP) by marine red alga, Corallina pilulifera methanol extract. Radiat Phys Chem. 2009;78:98-105.

83. Lawrence KP, Long PF, Young AR. Mycosporine-like amino acids for skin photoprotection. Curr Med Chem. 2018;25:5512-27.

84. Kohli I, Shafi R, Isedeh P, Griffith JL, Al-Jamal MS, Silpa-archa $\mathrm{N}$, et al. The impact of oral Polypodium leucotomos extract on ultraviolet B response: a human clinical study. J Am Acad Dermatol. 2017;77:33-41.e1.

85. Delgado-Wicke P, Rodríguez-Luna A, Ikeyama Y, Honma Y, Kume T, Gutierrez M, et al. Fernblock ${ }^{\circledR}$ upregulates NRF2 antioxidant pathway and protects keratinocytes from PM2.5-Induced xenotoxic stress. Oxid Med Cell Longev. 2020;2020:2908108.

86. Mohammad TF, Kohli I, Nicholson CL, Treyger G, Chaowattanapanit $\mathrm{S}$, Nahhas AF, et al. Oral polypodium leucotomos extract and its impact on visible light-induced pigmentation in human subjects. J Drugs Dermatol JDD. 2019;18:1198-203.

87. Torricelli P, Fini M, Fanti PA, Dika E, Milani M. Protective effects of Polypodium leucotomos extract against UVB-induced damage in a model of reconstructed human epidermis. Photodermatol Photoimmunol Photomed. 2017;33:156-63.

88. Emanuele E, Spencer JM, Braun M. An experimental double-blind irradiation study of a novel topical product (TPF 50) compared to other topical products with DNA repair enzymes, antioxidants, and growth factors with sunscreens: implications for preventing skin aging and cancer. J Drugs Dermatol. 2014;13:309-14. 